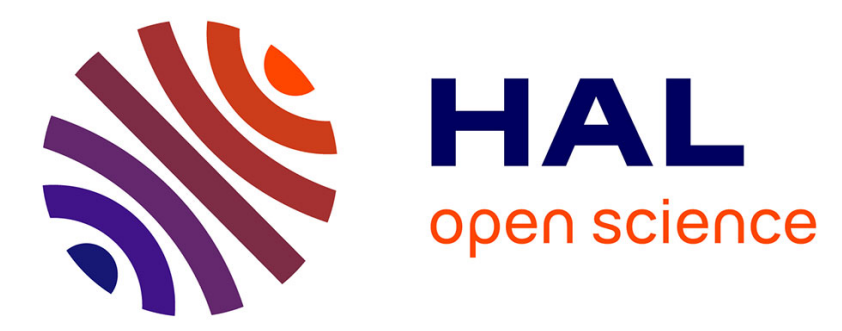

\title{
Continuum electromechanical theory for nematic continua with application to Freedericksz instability
}

Giampiero Pampolini, Nicolas Triantafyllidis

\section{To cite this version:}

Giampiero Pampolini, Nicolas Triantafyllidis. Continuum electromechanical theory for nematic continua with application to Freedericksz instability. 11e colloque national en calcul des structures, CSMA, May 2013, Giens, France. hal-01722107

\section{HAL Id: hal-01722107 https://hal.science/hal-01722107}

Submitted on 2 Mar 2018

HAL is a multi-disciplinary open access archive for the deposit and dissemination of scientific research documents, whether they are published or not. The documents may come from teaching and research institutions in France or abroad, or from public or private research centers.
L'archive ouverte pluridisciplinaire $\mathbf{H A L}$, est destinée au dépôt et à la diffusion de documents scientifiques de niveau recherche, publiés ou non, émanant des établissements d'enseignement et de recherche français ou étrangers, des laboratoires publics ou privés. 


\title{
Continuum electromechanical theory for nematic continua with appli- cation to Freedericksz instability
}

\author{
Giampiero PAMPOLINI ${ }^{1}$, Nicolas TRIANTAFYLLIDIS ${ }^{2}$
}

\author{
${ }^{1}$ Saint-Gobain Recherche, giampiero.pampolini@saint-gobain.com \\ ${ }^{2}$ Laboratoire de Mécanique des Solides Ecole Polytechnique, nick@1ms.polytechnique.fr
}

Résumé - In this communication we present the work by Pampolini and Triantafyllidis [1], in which an electro-mechanical theory for nematic continua is proposed. The theory is based on a variational approach and the equilibrium relations plus the Maxwell's equations are obtained as the Euler-Lagrange equations of a specific potential energy. The variational formulation is applied to the study of a 2D boundary value problem, termed in the literature as Freedericksz transition, where a nematic liquid crystal layer is confined between two plates and an electric field is applied perpendicular to the plates. This boundary value problem is treated as a bifurcation problem and an asymptotic analysis of the bifurcated equilibrium path is carried out.

Mots clés - nematic continua, electromechanical theory, variational approach, Freedericksz transition, asymptotic analysis

\section{Introduction}

Nematic elastomers are rubber-like solids formed by crosslinking polymeric chains that include liquid crystal molecules. In a simple description they consist of a network of polymeric strands connecting liquid crystal compartments of the size of some micrometers. The interaction between rubber elasticity of the network and the alignment of liquid crystal molecules leads to peculiar mechanical-optical [2] and electro-mechanical effects $[3,4]$.

One simple way to describe nematic ordering effects within a continuum theory is to represent the local molecular orientation through a unit vector, called the director. This idea was introduced by Oseen [5] and Zocher [6], further developed by Frank [7] and re-organized by Ericksen [8]. Terentjev [9] used the director description to model electro-mechanical effects in nematic elastomers by considering a total energy composed of three terms : the Frank-Oseen energy characteristic of liquid crystals, the energy of the electric field and an elastic term that models the resistance that the rubber network opposes to director rotation. Eringen [10] presented a thermo-electro-magnetic theory for liquid crystal polymers where stretching of liquid crystal molecules are taken into account by considering directors whose magnitudes change with motion. In the works by DeSimone et al. $[11,12,13]$ the electro-mechanical coupling effects of nematic elastomers were studied in the context of phase transforming materials by assuming a nonconvex strain energy density with multiple natural configurations .

This communication is based on the work by Pampolini and Triantafyllidis [1], in which an electromechanical theory for nematic continua is presented. Since the theory is based on recent variational formulations for electro-elastic solids [14, 15], it is suitable to deal with bifurcation problems and stability analysis of quasi-static evolutions. The outline of the present communication is as follows : in Section 2 we briefly describe the electromechanical theory. The Frank-Oseen director representation is adopted and a potential energy depending on four independent variables (the displacement, director, specific polarization and perturbed electric displacement potential) is constructed. The equilibrium plus the Maxwell's equations are thus obtained as the Euler-Lagrange equations of the potential energy. The theory can be used for both nematic elastomers and nematic liquid crystals depending on the choice of the free energy density.

In Section 3 a specific free energy of a nematic liquid crystal is considered and the variational formulation is applied to the analysis of a classical boundary value problem leading to a bifurcation. A 
infinite 2D layer of a nematic liquid crystal is confined between two parallel plates and is subjected to an electric field perpendicular to the plates. At the boundaries, the director lay parallel to the external plates. The remarkable behavior of liquid crystals originates from the competition between the alignment of the director prescribed at the boundary and the orientation favored by the electric field. For small electric fields, the director orientation is not influenced by the electric field. As the magnitude of the electric field overcomes a certain threshold value, the nematic begins to adjust its director orientation forwards the applied external electric field. This problem, known in the literature as splay Freedericksz transition, plays a basic role in determining the elastic properties of liquid crystals and it has many electro-optic applications [16].

\section{Electromechanical theory for nematic continua}

In this Section, we briefly present the electromechanical theory for nematic continua proposed in [1]. Following the Frank-Oseen model[5, 7], nematic ordering effects are described by using a unit vector $\mathbf{n}$, called the director, that represents the local molecular orientation. The specific (i.e. per unit mass) free energy $\Psi$ of the nematic continuum is taken to be function of the deformation gradient $\mathbf{F}$, the director $\mathbf{n}$, its gradient $\mathbf{n} \nabla$ and $\mathbf{p}$ the polarization per current volume ${ }^{1}$. The specific form of $\Psi$ depends on the application at hand.

We consider a nematic continuum of density $\rho$ occupying a volume $V$ and subjected to an externally applied electrical field $\mathbf{e}_{0}$ and to general mechanical loadings. The applied electric field is perturbed by the presence of the nematic continuum. To take into account this aspect, we define the total electric field e and the electric displacement $\mathbf{d}$ as follows (see [17]) :

$$
\mathbf{e}=\mathbf{e}_{0}+\widehat{\mathbf{e}}, \quad \mathbf{d}=\mathbf{d}_{0}+\widehat{\mathbf{d}}, \quad \mathbf{d}_{0}=\varepsilon_{0} \mathbf{e}_{0}, \quad \widehat{\mathbf{d}}=\varepsilon_{0} \widehat{\mathbf{e}}+\mathbf{p}, \quad \mathbf{x} \in V, \quad \widehat{\mathbf{d}}=\varepsilon_{0} \widehat{\mathbf{e}}, \quad \mathbf{x} \in \mathbb{R}^{3} \backslash V,
$$

where $\varepsilon_{0}$ is the electric permittivity of free space, $\mathbf{p}$ is the polarization per current volume and $\widehat{\mathbf{e}}, \widehat{\mathbf{d}}$ are the perturbed electric field and the perturbed electric displacement, respectively. Due to the absence of free electric charges, $\widehat{\mathbf{d}}$ can be represented in terms of a potential $\widehat{\mathbf{a}}$

$$
\widehat{\mathbf{d}}=\nabla \times \widehat{\mathbf{a}} .
$$

Assuming isothermal, quasistatic and non dissipative processes, the total stored energy of the system is the sum of the strain energy stored in the nematic continuum plus the electric energy stored in the entire space, namely

$$
\mathscr{E}=\int_{V} \rho \psi \mathrm{d} v+\int_{\mathbb{R}^{3}} \frac{\varepsilon_{0}}{2}(\widehat{\mathbf{e}} \bullet \widehat{\mathbf{e}}) \mathrm{d} v .
$$

Note that writing the electric energy in Eq.(3), we can take only the contribution due to the perturbed electric field, see [1] for justification. The potential of the externally applied loads is

$$
\mathscr{W}=-\int_{V}\left[\boldsymbol{\rho}(\mathbf{f} \bullet \mathbf{u}+\mathbf{g} \bullet \mathbf{n})+\mathbf{e}_{0} \bullet \mathbf{p}\right] \mathrm{d} v-\int_{\partial V}(\mathbf{t} \bullet \mathbf{u}+\mathbf{r} \bullet \mathbf{n}) \mathrm{d} a,
$$

where $\mathbf{f}, \mathbf{g}$ are respectively the body force and the body couple, both per unit mass, and $\mathbf{t}, \mathbf{r}$ are respectively the surface traction and the surface moment, both per unit current area da. By using Eqs. (1-4), the potential energy of the system $\mathscr{P}=\mathscr{E}+\mathscr{W}$ takes the form

$$
\mathscr{P}=\int_{V}\left[\rho(\psi-\mathbf{f} \bullet \mathbf{u}-\mathbf{g} \bullet \mathbf{n})-\mathbf{e}_{0} \bullet \mathbf{p}\right] \mathrm{d} v-\int_{\partial V}(\mathbf{t} \bullet \mathbf{u}+\mathbf{r} \bullet \mathbf{n}) \mathrm{d} a+\int_{\mathbb{R}^{3}} \frac{1}{2 \varepsilon_{0}}(\nabla \times \widehat{\mathbf{a}}-\mathbf{p}) \bullet(\nabla \times \widehat{\mathbf{a}}-\mathbf{p}) \mathrm{d} v .
$$

As it will be clear later, the electric field energy will give a Maxwell stress contribution to the total stress tensor in the mechanical equilibrium equations. To take into account for the Maxwell stress non linear dependence on electric field quantities, a Lagrangian formulation is necessary. For this purpose, we introduce the reference configuration electric displacement perturbation $\widehat{\mathbf{D}}$, its vector potential $\widehat{\mathbf{A}}$ and the per unit mass polarization $\mathbf{P}$ as follows :

$$
\widehat{\mathbf{D}}=J \mathbf{F}^{-1} \bullet \widehat{\mathbf{d}}, \quad \widehat{\mathbf{D}}=\nabla \times \widehat{\mathbf{A}}, \quad \mathbf{P}=\mathbf{p} / \rho,
$$

1. These quantities are function of the current position $\mathbf{x}$. In particular, $\mathbf{F} \equiv \mathbf{x} \nabla$ with $\nabla \equiv \partial / \partial \mathbf{X}$ is the gradient operator in the reference configuration while $\nabla \equiv \partial / \partial \mathbf{x}$ s the gradient operator in the current configuration 
where $J=\operatorname{det}(\mathbf{F})$. By using Eqs. (1) ${ }_{3}$ and (6) the perturbed electric field is written as function of $\widehat{\mathbf{A}}$ and $\mathbf{P}$ :

$$
\widehat{\mathbf{e}}=\frac{1}{\varepsilon_{0} J}\left(\mathbf{F} \bullet(\nabla \times \widehat{\mathbf{A}})-\rho_{0} \mathbf{P}\right) .
$$

where $\rho_{0}$ is the reference mass density defined on the entire space $\mathbb{R}^{3}$, e.g. $\rho_{0}(\mathbf{X}) \neq 0$ for $\mathbf{X} \in V$ while $\rho_{0}(\mathbf{X})=0$ for $\mathbf{X} \in \mathbb{R}^{3} \backslash V$. The system's potential energy, in reference configuration field quantities, takes the form

$$
\begin{aligned}
\mathscr{P}=\int_{V} \rho_{0}\left(\psi-\mathbf{f} \bullet \mathbf{u}-\mathbf{g} \bullet \mathbf{n}-\mathbf{e}_{0} \bullet \mathbf{P}\right) \mathrm{d} V & -\int_{\partial V}(\mathbf{T} \bullet \mathbf{u}+\mathbf{R} \bullet \mathbf{n}) \mathrm{d} A \\
& +\int_{\mathbb{R}^{3}} \frac{1}{2 \varepsilon_{0} J}\left(\mathbf{F} \bullet(\nabla \times \widehat{\mathbf{A}})-\rho_{0} \mathbf{P}\right) \bullet\left(\mathbf{F} \bullet(\nabla \times \widehat{\mathbf{A}})-\rho_{0} \mathbf{P}\right) \mathrm{d} V
\end{aligned}
$$

where $\mathbf{T}, \mathbf{R}$ are the reference counterparts of the traction $\mathbf{t}$ and moment $\mathbf{r}$, respectively ${ }^{2}$.

The potential energy in (8) depends on four independent variables : the displacement $\mathbf{u}=\mathbf{X}-\mathbf{x}$, the director $\mathbf{n}$, the specific polarization $\mathbf{P}$ and the electric displacement perturbation potential $\widehat{\mathbf{A}}$. Variations of $\mathscr{P}$ with respect to each one of these independent variables lead to the governing Euler-Lagrange equations and associated boundary/interface conditions. In particular, variation of $\mathscr{P}$ with respect $\mathbf{n}$ gives the director equilibrium and the corresponding interface condition, namely

$$
\begin{array}{ll}
\nabla \bullet\left(\frac{\partial \psi}{\partial(\mathbf{n} \nabla)}\right)^{\mathrm{T}}-\frac{\partial \psi}{\partial \mathbf{n}}+\mathbf{g}=\mathbf{0}, & \mathbf{X} \in V, \\
\mathbf{N} \bullet\left[\left[\frac{\partial \psi}{\partial(\mathbf{n} \nabla)}\right]\right]=\mathbf{R}, & \mathbf{X} \in \partial V,
\end{array}
$$

where $\mathbf{N}$ is the outward normal to $\partial V^{3}$

When $\mathscr{P}$ is extremized with respect to $\widehat{\mathbf{A}}$, the Euler-Lagrange equations give the Faraday's law and the associated interface condition

$$
\begin{array}{lc}
\nabla \times \widehat{\mathbf{E}}=\mathbf{0}, & \mathbf{X} \in \mathbb{R}^{3}, \\
\mathbf{N} \times[\widehat{\mathbf{E}}]=\mathbf{0}, & \mathbf{X} \in \partial V, \\
\widehat{\mathbf{E}} \equiv \frac{1}{\varepsilon_{0} J}\left(\mathbf{F} \bullet(\nabla \times \widehat{\mathbf{A}})-\rho_{0} \mathbf{P}\right) \bullet \mathbf{F} & =\widehat{\mathbf{e}} \bullet \mathbf{F},
\end{array}
$$

The derivation of $\mathscr{P}$ with respect to the specific polarization $\mathbf{P}$ results in the electric part of the constitutive equation

$$
\frac{\partial \psi}{\partial \mathbf{P}}=\mathbf{e}
$$

and the derivation with respect to the displacement $\mathbf{u}$ yelds

$$
\begin{array}{lc}
\widehat{\boldsymbol{\Pi}}=\boldsymbol{\Pi}_{S}+\widehat{\boldsymbol{\Pi}}_{M}, \quad \boldsymbol{\Pi}_{S}=\rho_{0}\left(\frac{\partial \psi}{\partial \mathbf{F}}\right)^{\mathrm{T}}, & \\
\widehat{\boldsymbol{\Pi}}_{M}=\frac{1}{\varepsilon_{0} J}\left[(\nabla \times \widehat{\mathbf{A}}) \otimes\left(\mathbf{F} \bullet(\nabla \times \widehat{\mathbf{A}})-\rho_{0} \mathbf{P}\right)-\frac{1}{2}\left\|\mathbf{F} \bullet(\nabla \times \widehat{\mathbf{A}})-\rho_{0} \mathbf{P}\right\|^{2} \mathbf{F}^{-1}\right], \\
\nabla \bullet \widehat{\Pi}+\rho_{0}\left(\mathbf{f}+\mathbf{P} \bullet\left(\mathbf{e}_{0} \nabla\right) \bullet \mathbf{F}^{-1}\right)=\mathbf{0}, & \mathbf{X} \in \mathbb{R}^{3}, \\
\mathbf{N} \bullet[[\widehat{\boldsymbol{\Pi}}]]=\mathbf{T}, & \mathbf{X} \in \partial V,
\end{array}
$$

Note that the perturbed stress tensor $\widehat{\Pi}$ is the sum of the nematic constitutive part $\Pi_{S}$ and the perturbed Maxwell stress $\widehat{\boldsymbol{\Pi}}_{M}$. Equilibrium equations that involve only mechanical body forces are obtained when $\widehat{\Pi}$ is replaced by a total stress tensor, defined as the sum of the $\Pi_{S}$ and a total Maxwell stress, see [1] for details.

2. Henceforth quantities associated with the reference configuration are denoted by capital symbols to distinguish them from their current configuration counterparts

3. $\mathbf{N}$ the outward normal to $\partial V$ is not to be confused with the nematic director $\mathbf{n}(\mathbf{X})$. 


\section{The 2D boundary value problem - The Freedericksz transition}

\subsection{Material selection}

To represent an incompressible nematic liquid crystal, the free energy $\psi$ is taken to be the sum of two contributions : the Frank-Oseen energy $\psi^{F-O}$ characteristic of nematics ${ }^{4}$, and a polarization energy $\psi^{P}$

$$
\begin{aligned}
& \rho \psi(\mathbf{n}, \mathbf{n} \nabla, \mathbf{P})=\rho \psi^{F-O}(\mathbf{n}, \mathbf{n} \nabla)+\rho \psi^{P}(\mathbf{n}, \mathbf{P}), \\
& \rho \psi^{F-O}=\frac{1}{2} k_{1}(\nabla \bullet \mathbf{n})^{2}+\frac{1}{2} k_{2}(\mathbf{n} \bullet(\nabla \times \mathbf{n}))^{2}+\frac{1}{2} k_{3}\|\mathbf{n} \times(\nabla \times \mathbf{n})\|^{2}+\frac{1}{2} c_{1}(\mathbf{n} \bullet \mathbf{n}-1)^{2}, \\
& \rho \psi^{P}=\frac{\rho^{2}}{2 \varepsilon_{0}}\left(\chi^{-1} \mathbf{P} \bullet \mathbf{P}+\left(\chi_{n}^{-1}-\chi^{-1}\right)(\mathbf{P} \bullet \mathbf{n})^{2}\right),
\end{aligned}
$$

where $k_{1}, k_{2}$ and $k_{3}$ are positive constants, called in the literature the splay, twist and bend constants, respectively. The inextensibility of the director $\mathbf{n}$ is taken into account by the penalization term $(\mathbf{n} \boldsymbol{n}-1)^{2}$ multiplied by a large constant $c_{1}$. In (13) $\chi$ and $\chi_{n}$ are the electric susceptibility constants when the electric field and the director $\mathbf{n}$ are perpendicular or parallel, respectively.

\subsection{Problem description and bifurcation analysis}

Consider a two-dimensional infinite layer of a nematic liquid crystal confined between two parallel plates at distance $l$. An electric field $e_{0}$ is applied perpendicular to the plates. The director $\mathbf{n}$ is anchored parallel to the plates at the extremities, so the essential boundary conditions are

$$
n_{1}\left(x_{1}, \pm l / 2\right)=1, \quad n_{2}\left(x_{1}, \pm l / 2\right)=0 .
$$

For every value of the applied electric field the equilibrium configuration can be found by extremizing the potential energy $\mathscr{P}$,

$$
\mathscr{P}, \mathbf{v}(\mathbf{v}) \delta \mathbf{v}=0, \quad \mathbf{v} \equiv(\mathbf{n}, \mathbf{P}, \alpha) .
$$

Note that we neglect the variation with respect to $\mathbf{u}$ because the liquid crystal is incompressible and there is not interest in computing forces ${ }^{5}$. One obvious solution to equation (15) is the principal solution, which corresponds to constant director orientation, namely

$$
\stackrel{0}{\mathbf{v}}=\left\{\begin{array}{l}
0 \\
\mathbf{n}=(1,0) \\
\stackrel{0}{\rho}=\left(0, \frac{\chi}{1+\chi} \varepsilon_{0} e_{0}\right) \\
0 \\
\alpha=0
\end{array}\right.
$$

At small values of the applied electric field, the principal solution $\mathbf{v}_{\mathbf{v}}$ is stable. As $e_{0}$ increases, it reaches a value $e_{0}^{c}$ where the principal solution is no longer a minimizer of the potential energy, but where the energy vanishes along a particular direction $\mathbf{v}$, called the critical mode, which satisfies the condition :

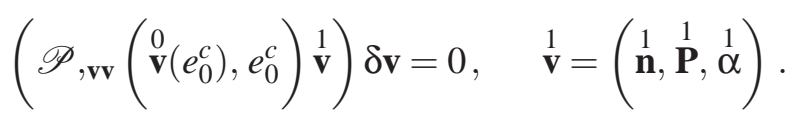

Eq. (17) can be transformed in a system of three partial differential equations in terms of $\mathbf{n}=\left(n_{1}, n_{2}\right)$

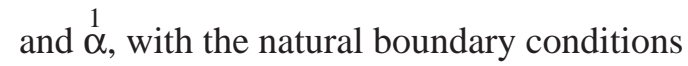

$$
\stackrel{1}{\alpha}, 2\left(x_{1}, \pm l / 2\right)=0
$$

These natural boundary conditions are compatible with a fixed voltage at the end plates, as discussed in [1].

4. The saddle splay term of the Frank-Oseen energy is here omitted since it does not contribute to the bulk energy, see [18] for details.

5. We take $\mathbf{F}=\mathbf{I}$ 


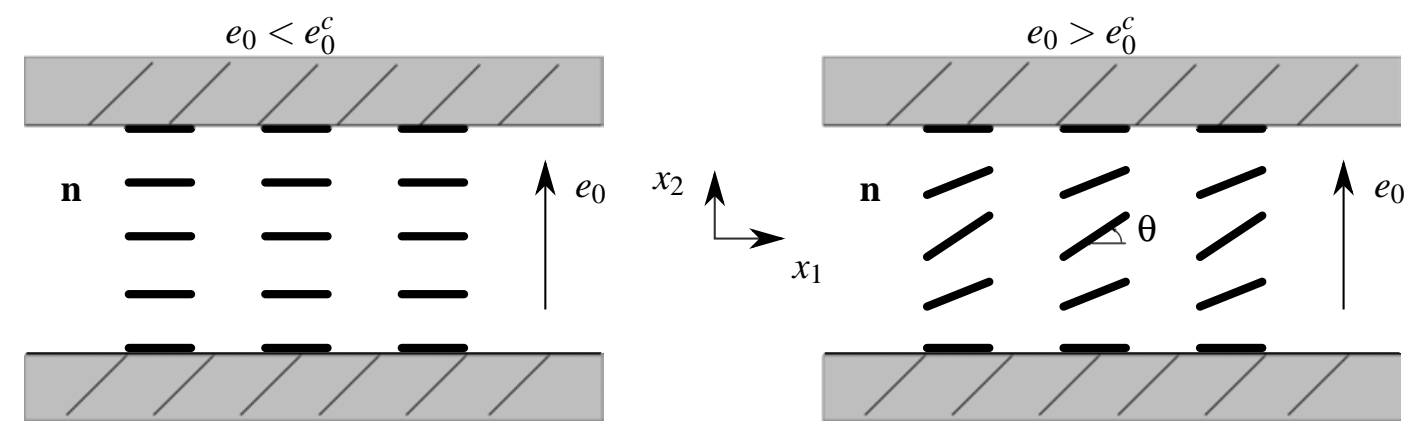

Fig. 1 - Principal solution for $e_{0}<e_{0}^{c}$ (left) and bifurcated solution for $e_{0}>e_{0}^{c}$ (right).

The symmetries in the problem allow for the following Fourier decomposition with respect the $x_{1}$-direction of the critical mode $\mathbf{v}$,

$$
\mathscr{S}^{1}:\left\{\begin{array}{l}
\stackrel{1}{n}_{1}=n_{1}\left(x_{2}\right) \sin \left(\omega_{1} x_{1}\right), \\
1 \\
n_{2}=-n_{2}\left(x_{2}\right) \cos \left(\omega_{1} x_{1}\right), \\
1 \\
\alpha=\alpha\left(x_{2}\right) \sin \left(\omega_{1} x_{1}\right),
\end{array} \quad \mathscr{A}^{1}:\left\{\begin{array}{l}
1 \\
n_{1}=n_{1}\left(x_{2}\right) \cos \left(\omega_{1} x_{1}\right), \\
1 \\
n_{2}=n_{2}\left(x_{2}\right) \sin \left(\omega_{1} x_{1}\right), \\
1 \\
\alpha=-\alpha\left(x_{2}\right) \cos \left(\omega_{1} x_{1}\right),
\end{array}\right.\right.
$$

where the symbols $\mathscr{S}^{1}$ and $\mathscr{A}^{1}$ denote the symmetric and antisymmetric parts of the mode. By assuming the director to be inextensible, one obtains $n_{1}=0$, and the following expressions for $n_{2}\left(x_{2}\right)$ and $\alpha\left(x_{2}\right)$ (see [1]) :

$$
\mathscr{S}^{2}:\left\{\begin{array}{l}
n_{2}=V_{n} \cosh \left(\omega_{2} x_{2}\right), \\
\alpha=V_{\alpha} \sinh \left(\omega_{2} x_{2}\right),
\end{array} \quad \mathscr{A}^{2}:\left\{\begin{array}{l}
n_{2}=V_{n} \sinh \left(\omega_{2} x_{2}\right), \\
\alpha=V_{\alpha} \cosh \left(\omega_{2} x_{2}\right),
\end{array}\right.\right.
$$

where the symbols $\mathscr{S}^{2}$ and $\mathscr{A}^{2}$ denote the symmetric and antisymmetric parts with respect to the coordiante $x_{2}$. The constants $\omega_{2}, V_{n}$ and $V_{\alpha}$ entering (20) are related by

$$
\begin{gathered}
\mathbf{Q}\left(\omega_{1}, \omega_{2}\right) \bullet \mathbf{V}=0 \\
\mathbf{Q} \equiv\left[\begin{array}{cc}
k_{3}\left(\omega_{1}\right)^{2}-k_{1}\left(\omega_{2}\right)^{2}-\varepsilon_{0} \bar{\chi}\left(e_{0}^{c}\right)^{2} & \bar{\chi} e_{0}^{c} \omega_{2} \\
\bar{\chi} e_{0}^{c} \omega_{2} & \frac{1}{\varepsilon_{0}(1+\chi)}\left(\omega_{2}\right)^{2}-\frac{1}{\varepsilon_{0}\left(1+\chi_{N}\right)}\left(\omega_{1}\right)^{2}
\end{array}\right], \quad \mathbf{V} \equiv\left[\begin{array}{c}
V_{n} \\
V_{\alpha}
\end{array}\right],
\end{gathered}
$$

with $\bar{\chi} \equiv\left(\chi_{N}-\chi\right) /\left[(1+\chi)\left(1+\chi_{N}\right)\right]$ and $\omega_{2}$ is the solution of the bi-quadratic :

$$
\operatorname{det}\left(\mathbf{Q}\left(\omega_{1}, \omega_{2}\right)\right)=0 .
$$

It is possible to show that the boundary conditions (14) and (18) enforce $\omega_{2}$ to be a pure imaginary number with values

$$
\mathscr{S}^{2}: \omega_{2}=i(2 m-1) \pi / l, \quad \mathscr{A}^{2}: \omega_{2}=i 2 m \pi / l .
$$

A detailed derivation of this result is given in [1].

The critical electric field $e_{0}^{c}$ is then found as the minimum value of $e_{0}$ that satisfies (22). By using (23) in (22), one shows that the lowest $e_{0}^{c}$ correspond to $\omega_{1}=0, \omega_{2}=i \pi / l$ (i.e. $m=1$ ), and it takes the value

$$
e_{0}^{c}=\frac{\pi}{l}(1+\chi)\left(\frac{k_{1}}{\varepsilon_{0}\left(\chi_{n}-\chi\right)}\right)^{1 / 2} .
$$

The total critical electric field in the $x_{2}$-direction $e^{c}$ is the sum of the externally applied field $e_{0}^{c}$ plus the perturbed electric field $\widehat{e}^{c}=-e_{0}^{c} \chi /(1+\chi)$, and by using (24) we have $e^{c}=(\pi / l)\left[k_{1} / \varepsilon_{0}\left(\chi_{n}-\chi\right)\right]^{1 / 2}$ which is exactly the value stated in [18] in his Eq.(3.208). 
Finally in view of $\omega_{1}=0$ and $\omega_{2}=i \pi / l$, the critical mode $\mathbf{v}$ takes the form

$$
\stackrel{1}{\mathbf{v}}=\left\{\begin{array}{l}
\frac{1}{\mathbf{n}}=\left(0, \cos \left(\pi x_{2} / l\right)\right), \\
\rho \mathbf{P}=\left(\left(k_{1} \varepsilon_{0}\left(\chi_{n}-\chi\right)\right)^{1 / 2} \cos \left(\pi x_{2} / l\right), 0\right), \\
\stackrel{1}{\alpha}=\frac{l}{\pi}\left(k_{1} \varepsilon_{0}\left(\chi_{n}-\chi\right)\right)^{1 / 2} \sin \left(\pi x_{2} / l\right),
\end{array}\right.
$$

\subsection{LSK asymptotic analysis}

It is typical of most boundary value problems exhibiting bifurcations that a post-bifurcated solution has no easily obtainable analytic solution. To remedy this situation, an asymptotic analysis of the problem is possible that provides the initial dependence of the critical load and corresponding eigenmode on the bifurcation amplitude.

This asymptotic technique, termed "Lyapunov - Schmidt - Koiter" method (LSK) is applied here to determine the bifurcated equilibrium solution near the critical point and check its stability. According to the general theory presented in [19], the asymptotic expansion for the applied electric field $e_{0}$ and the bifurcated equilibrium solution $\mathbf{v}$ about the critical point $e_{0}^{c}$ are given by

$$
e_{0}=e_{0}^{c}+\xi e_{0}^{1}+\frac{\xi^{2}}{2} e_{0}^{2}+\mathrm{O}\left(\xi^{3}\right), \quad \mathbf{v}=\stackrel{0}{\mathbf{v}}\left(e_{0}^{c}\right)+\xi_{\mathbf{v}}^{1}+\frac{\xi^{2}}{2} \stackrel{2}{\mathbf{v}}+\mathrm{O}\left(\xi^{3}\right)
$$

with $\xi$ the "bifurcation amplitude parameter" defined as the projection of the bifurcated solution on the eigenmode $\mathbf{v}$, namely

$$
\xi \equiv\langle\mathbf{v}-\mathbf{v}, \mathbf{v}\rangle=\frac{2}{|A|} \int_{A}\left(n_{i}-n_{i}\right) n_{i} \mathrm{~d} A, .
$$

where $|A|$ denotes the area of the domain $A$. A remark is in order at this point to justify the choice of the norm used in Eq. (27). Usually in the literature, the bifurcated solution is computed by introducing the director distortion angle $\theta$, see Fig.1. The chosen norm gives a direct relation between the amplitude of the mode $\xi$ and the director distortion angle $\theta$, e.g. $\xi=\sin \theta(0)$. The norm of the eigenmode $\mathbf{v}$ is assumed to be unity and the expressions in (25) reflect this assumption.

The bifurcation point of $e_{0}^{c}$ is a simple one, since the eigenmode $\mathbf{v}$ in (25) is unique (up to an amplitude), see [19] for details. Moreover the bifurcation is a symmetric one since

$$
\mathscr{P}_{, \mathbf{v v}}^{c} \mathbf{V} \mathbf{V} \mathbf{V} \mathbf{V}=0 .
$$

Consequently in (26) ${ }_{1} e_{0}^{1}=0$, the first term in the asymptotic expansion of the applied electric field vanishes and the calculation of $e_{0}^{2}$ requires the calculation of $\mathbf{v}$ the second order term in the expansion $(26)_{2}$, which is obtained by the solution of the following variational equation

$$
\left(\mathscr{P}_{, \mathbf{v v}}^{c} \mathbf{v}+\left(\mathscr{P}_{, \mathbf{v v v}}^{c} \mathbf{v}\right) \mathbf{v}\right) \delta \mathbf{v}=0, \quad \text { with } \quad\langle\delta \mathbf{v}, \mathbf{v}\rangle=0
$$

Making use of (25), it can be shown that the solution of the equation (29) results in the following expression for $\mathbf{v}^{2}$ :

$$
\stackrel{2}{\mathbf{v}}=\left\{\begin{array}{l}
\stackrel{2}{\mathbf{n}}=\left(-\cos ^{2}\left(\pi x_{2} / l\right), 0\right), \\
\stackrel{2}{\mathbf{P}}=\left(0, \frac{2 \pi}{(1+\chi) l}\left[k_{1} \varepsilon_{0}\left(\chi_{n}-\chi\right)\right]^{1 / 2} \cos ^{2}\left(\pi x_{2} / l\right)\right), \\
\stackrel{2}{\alpha}=0 .
\end{array}\right.
$$


Tableau 1 - Coefficients of a typical nematic liquid crystal used in the numerical calculations (see [18])

\begin{tabular}{lccr}
\hline$k_{1}[\mathrm{~N}]$ & $k_{3}[\mathrm{~N}]$ & $\chi$ & $\chi_{n}$ \\
\hline $6.2 \times 10^{-12}$ & $8.2 \times 10^{-12}$ & 6 & 17.5 \\
\hline
\end{tabular}

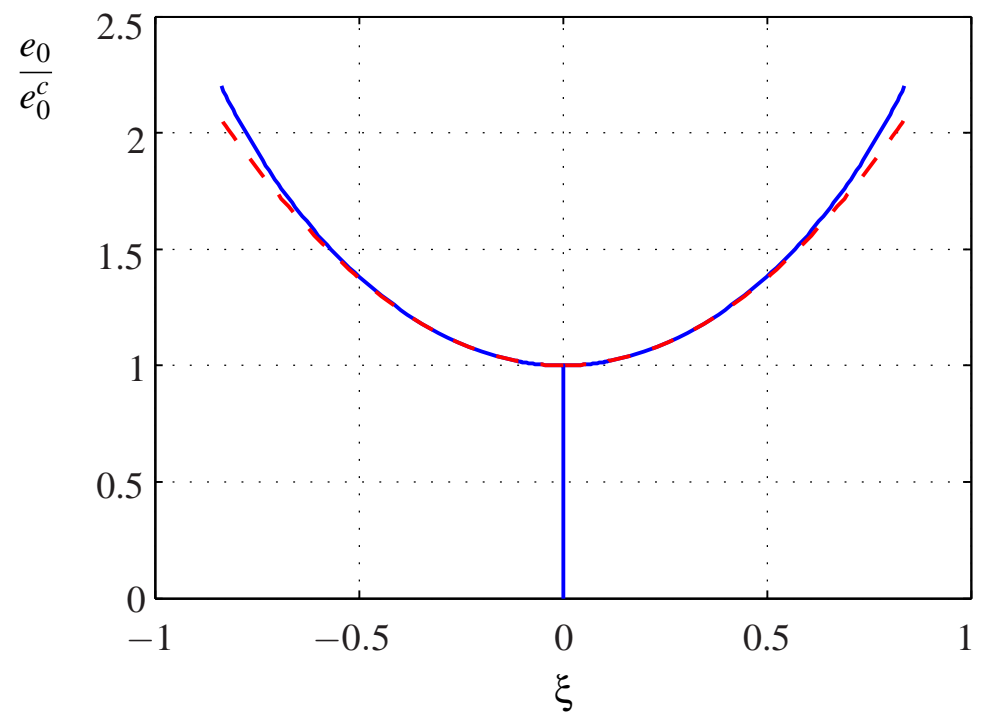

Fig. 2 - Graph of external electric field versus the amplitude $\xi$ of the bifurcated mode for the exact solution (full line) and for the asymptotic solution (dotted line).

The first non zero coefficient in the asymptotic expansion of $e_{0}$ can now be calculated from the general theory [19] using ${ }_{\mathbf{v}}^{2}$ in (30) from the following expression

$$
e_{0}^{2}=-\frac{1}{3} \frac{\left(\left(\left(\mathscr{P}_{, \mathbf{v v v v}}^{c} \mathbf{v}\right) \mathbf{v}\right) \mathbf{v}\right) \mathbf{v}+3\left(\left(\mathscr{P}_{, \mathbf{v v v}}^{c} \mathbf{v}\right) \mathbf{v}\right) \mathbf{v}}{\left(\left(\mathrm{d} \mathscr{P}_{, \mathbf{v v}}^{c} / \mathrm{d} e_{0}\right)^{c} \mathbf{v}\right) \mathbf{v}} .
$$

The stability of the bifurcated equilibrium path in the neighborhood of $e_{0}^{c}$ depends on the sign of $e_{0}^{2}$; if $e_{0}^{2}>0$ the bifurcated path is stable, it minimizes the potential energy $\mathscr{P}$ in a neighborhood of the critical point, while for $e_{0}^{2}<0$ it is unstable near the critical point. Upon using relations (25) and (30) into (31), the coefficient $e_{0}^{2}$ is found to be :

$$
e_{0}^{2}=\frac{3 k_{1}\left(\chi_{n}-\chi\right)+k_{3}(1+\chi)}{2\left(k_{1} \varepsilon_{0}\left(\chi_{n}-\chi\right)\right)^{1 / 2}} \frac{\pi}{l}>0
$$

The stability of the bifurcated path near $e_{0}^{2}$ follows, since a bifurcated solution exists only when $\chi_{n}>\chi$. The details of these asymptotic calculations are given in [1]. In Fig.2 the asymptotic solution id compared with the exact bifurcated solution, given in the literature [18], for a typical nematic liquid crystal with material parameters given in Table 1.

\section{Références}

[1] G. Pampolini and N. Triantafyllidis. Continuum electromechanical theory for nematic continua with application to freedericksz instability. submitted, 2013.

[2] M. Warner and E.M. Terentjev. Liquid crystal elastomers. Clarendon Press Oxford, 2007.

[3] Yusril Yusuf, Jong-Hoon Huh, P. E. Cladis, Helmut R. Brand, Heino Finkelmann, and Shoichi Kai. Low-voltage-driven electromechanical effects of swollen liquid-crystal elastomers. Phys. Rev. E, $71: 061702$, Jun 2005. 
[4] K. Urayama and T. Takigawa. Electromechanical effects in swollen nematic elastomers. In D.J. Broer, G.P. Crawford, and S. Zumer, editors, Cross-linked liquid crystalline systems : From rigid polymer networks to elastomers, Liquid Crystals Book Series, pages 473-486. CRC Press by Taylor \& Francis Group, 2011.

[5] C. W. Oseen. The theory of liquid crystals. Trans. Faraday Soc., 29(140) :883-899, 1933.

[6] H. Zocher. The effect of a magnetic field on the nematic state. Trans. Faraday Soc., 29(140) :945$957,1933$.

[7] F. C. Frank. On the theory of liquid crystals. Discuss. Faraday Soc., $25: 19-28,1958$.

[8] J. L. Ericksen. Hydrostatic theory of liquid crystals. Archive for Rational Mechanics and Analysis, 9(1) :371-378, 1962.

[9] E. M. Terentjev, M. Warner, R. B. Meyer, and J. Yamamoto. Electromechanical fredericks effects in nematic gels. Phys. Rev. E, $60: 1872-1879$, Aug 1999.

[10] A. C. Eringen. A unified continuum theory for electrodynamics of polymeric liquid crystals. International Journal of Engineering Science, 38(9-10):959 - 987, 2000.

[11] A. DeSimone, A. DiCarlo, and L. Teresi. Critical voltages and blocking stresses in nematic gels. The European Physical Journal E : Soft Matter and Biological Physics, 24(3) :303-310, 2007.

[12] A. Fukunaga, K. Urayama, T. Takigawa, A. DeSimone, and L. Teresi. Dynamics of electro-optomechanical effects in swollen nematic elastomers. Macromolecules, 41(23) :9389-9396, 2008.

[13] P. Cesana and A. DeSimone. Strain-order coupling in nematic elastomers : equilibrium configurations. Math. Models Methods Appl. Sci, 19 :601-630, 2009.

[14] J. L. Ericksen. Theory of elastic dielectrics revisited. Archive for Rational Mechanics and Analysis, 183(2) :299-313, 2007.

[15] R. Bustamante, A. Dorfmann, and R. Ogden. Nonlinear electroelastostatics : a variational framework. Zeitschrift für Angewandte Mathematik und Physik (ZAMP), 60 :154-177, 2009.

[16] P.G. de Gennes and J. Prost. The physics of liquid crystals. Clarendon Press Oxford, 1993.

[17] R. A. Toupin. The elastic dielectric. J. Rational Mech. Anal, 5(6) :849-915, 1956.

[18] I. W. Stewart. The static and dynamic continuum theory of liquid crystals : a mathematical introduction. Liquid Crystals Book Series. Taylor \& Francis, 2004.

[19] N. Triantafyllidis and R. Peek. Worst shapes of imperfections for space trusses with many simultaneously buckling members. International Journal of Solids and Structures, 29(19):2385-2402, 1992. 